\title{
Uso del ATC en la Elefantisis nostras verrucosa colonizada
}

\author{
Armengol-de-la-Hoz J.M. ${ }^{1}$, Martínez-Murillo A. ${ }^{2}$, Espejo-Ortega L.F. ${ }^{3}$
}

Sanid. mil. 2021; 77 (1): 26-28, ISSN: 1887-8571

\section{RESUMEN}

La Elefantiasis nostras verrucosa es una rara afectación dermatológica caracterizada por edema e hiperqueratosis maloliente con liquenificación generalizada, pápulas adoquinadas y cambios verrugosos. La patología es producida por un linfedema obstructivo crónico congénito o secundario. No existe un consenso claro para el tratamiento de esta patología, en este artículo presentamos un caso clínico de una paciente de 73 años que fue tratada con ácido tricloracético al $25 \%$ con resultados muy satisfactorios.

PALABRAS CLAVE: Linfedema, Elefantiasis nostras verrucosa, ácido tricloracético, miasis.

\section{The treatment of Elephantiasis nostras verrucosa with TCA}

\section{SUMMARY}

Elephantiasis nostras verrucosa is a rare dermatological condition characterized by edema, foul-smelling hyperkeratosis with generalized lichenification, cobblestone papules, and warty changes. The pathology is produced by congenital or secondary chronic obstructive lymphedema. There is no clear consensus for the treatment of this pathology. In this article we present a clinical case of a 73 years old woman who was treated with $25 \%$ trichloracetic acid with satisfactory results.

KEYWORDS: Lymphedema, Elephantiasis nostras verrucosa, trichloracetic acid, myiasis.

\section{INTRODUCCIÓN}

La Elefantiasis nostras verrucosa (ENV) es una complicación rara del linfedema crónico que exige de una larga evolución para producirse ${ }^{1}$. Este linfedema puede ser congénito o secundario a una infección no filariásica (tuberculosis, micosis, sífilis), cirugía, radioterapia, traumatismo, obstrucción neoplásica, obesidad, hipertensión portal o insuficiencia cardíaca congestiva ${ }^{2,3}$.

Con respecto al tratamiento de la ENV, existen numerosos trabajos describiendo desde tratamientos quirúrgicos agresivos ${ }^{4}$, hasta enfoques más conservadores ${ }^{5,6}$ pero ninguno ha demostrado una clara superioridad, lo que deja al facultativo en la necesidad de ponderar cuál es el tratamiento más adecuado en función de las características de cada paciente. En este caso clínico describimos el uso del ácido tricloracético (ATC) para el tratamiento de esta rara patología.

\section{CASO CLÍNICO}

Presentamos el caso de una paciente mujer de 73 años de edad sin alergias conocidas ni antecedentes de interés que ingresó en el servicio de Medicina Intensiva por un cuadro de shock séptico no filiado con fracaso multiorgánico. Asimismo, la paciente presentaba en los miembros inferiores unas

\footnotetext{
${ }^{1}$ Teniente Médico. Escuela Militar de Sanidad (EMISAN). Academia Central de la Defensa (ACD). Madrid.

${ }^{2}$ Teniente Coronel Médico. Servicio Cirugía Plástica, Estética y Reparadora. Hospital Central de la Defensa «Gómez Ulla». Madrid.

${ }^{3}$ Coronel Médico. Jefe de Servicio. Cirugía Plástica, Estética y Reparadora. Hospital Central de la Defensa «Gómez Ulla». Madrid.
}

Recibido: 30 de octubre de 2020

Aceptado: 20 de diciembre de 2020

doi: $10.4321 /$ S1887-85712021000100004 lesiones hiperqueratósicas malolientes con liquenificación generalizada y lo que aparentaban ser hifas por sobrecrecimiento fúngico (Figura 1).

Tras la inicial estabilización hemodinámica, se procedió a realizar un diagnóstico diferencial de las posibles causas del shock, estableciéndose las lesiones en miembros inferiores como origen del mismo. En la valoración primaria de las lesiones en las extremidades inferiores se evidenció una colonización de las lesiones por vermes, los cuales fueron remitidos para estudio al servicio de Microbiología donde se filiaron como larvas de musca domestica (Figura 2).

Tras la valoración del caso por el servicio de Cirugía Plástica, Estética y Reparadora se confirmó el diagnóstico de ENV y se estableció como tratamiento la realización de curas diarias

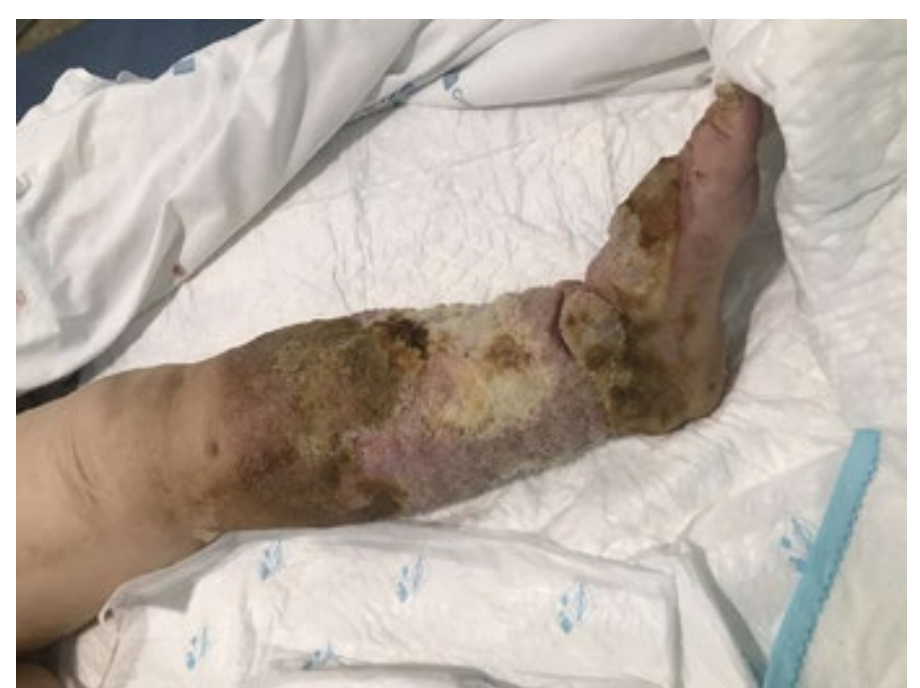

Figura 1. Primer día de ingreso, lesiones hiperqueratósicas con liquenificación generalizada. 


\section{Uso del ATC en la Elefantisis nostras verrucosa colonizada}

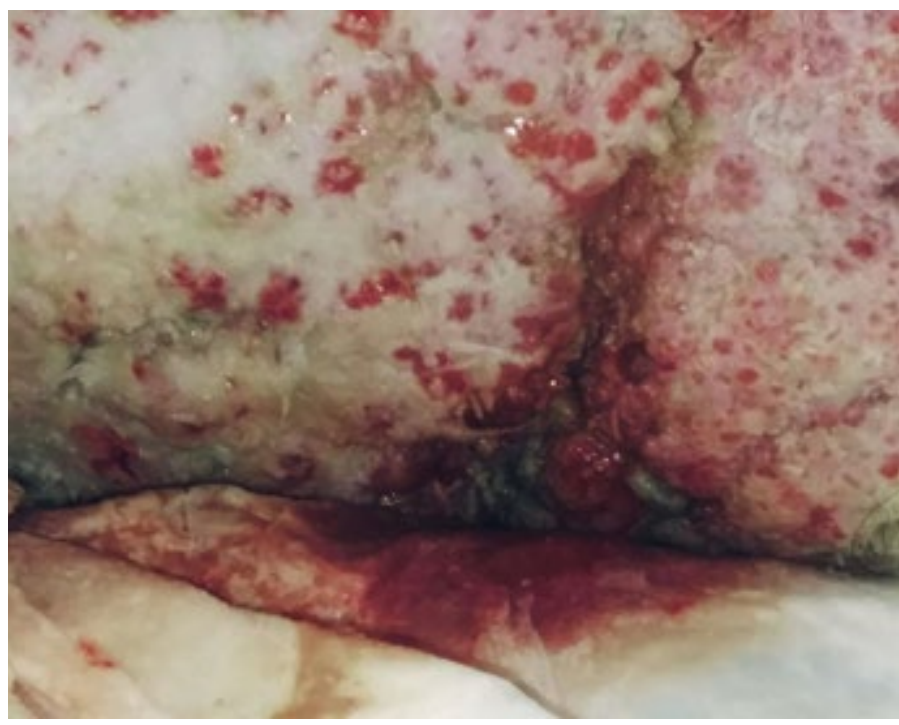

Figura 2. Larvas de musca domestica en las lesiones de la paciente.

con ATC al 25\% para la eliminación de la capa hiperqueratósica que cubría la piel.

Para realizar las curas se utilizaron compresas impregnadas en el ATC al 25\% y se cubrieron las lesiones durante 24 horas con un vendaje oclusivo. Las áreas de piel sana se protegieron con una pomada de mupirocina evitando así su lesión y como profilaxis de sobreinfección.

Pasadas veinticuatro horas del inicio del tratamiento, donde no se constató movimiento de larvas en la lesión, se realizó desbridamiento con una esponja de clorhexidina de manera agresiva eliminando parte de la zona hiperqueratósica y numerosas larvas.

Tres días después, desaparecieron por completo las larvas y la zona de la piel hiperqueratósica empezó a desprenderse (Figura 3). El desbridamiento y la aplicación del ATC se realizó con una frecuencia diaria hasta que desapareció toda la capa que presentaba esta queratosis.

La paciente evolucionó favorablemente del cuadro de shock séptico y pudo ser trasladada a la planta de hospitalización donde se continuó con el tratamiento-

Tras 52 días de ingreso hospitalario, la paciente fue dada de alta objetivándose la resolución completa de las lesiones en miembros inferiores (Figura 4).

\section{DISCUSIÓN}

La primera descripción en la historia de un caso de ENV pudo ser realizada por Celso (30 a.C.-50 d.C.) quién describió una patología en la que el paciente presentaba una lesión cutánea en miembros inferiores que la asemejaba a la pezuña de un elefante $^{7}$. En los siglos posteriores se siguieron describiendo casos de elefantiasis asociadas a cuadros infecciosos, especialmente filariasis y lepra.

En los primeros años de siglo XX, William Halsted en Baltimore, describe los primeros casos de elefantiasis postquirúrgica tras mastectomía con vaciamiento ganglionar axilar, que denominaría elephantiasis chirurgica ${ }^{8}$.

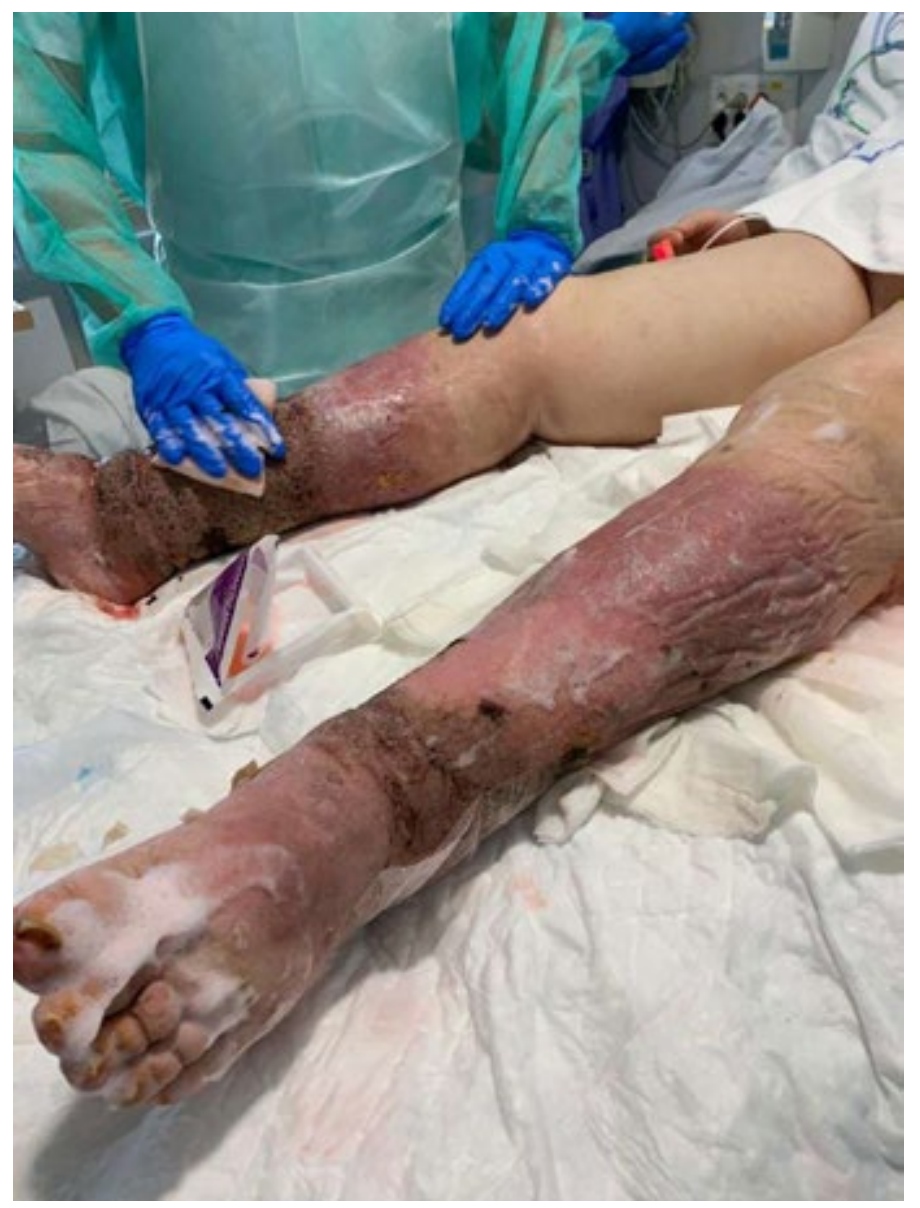

Figura 3. Mejoría de las lesiones, proceso de desbridamiento mecánico.

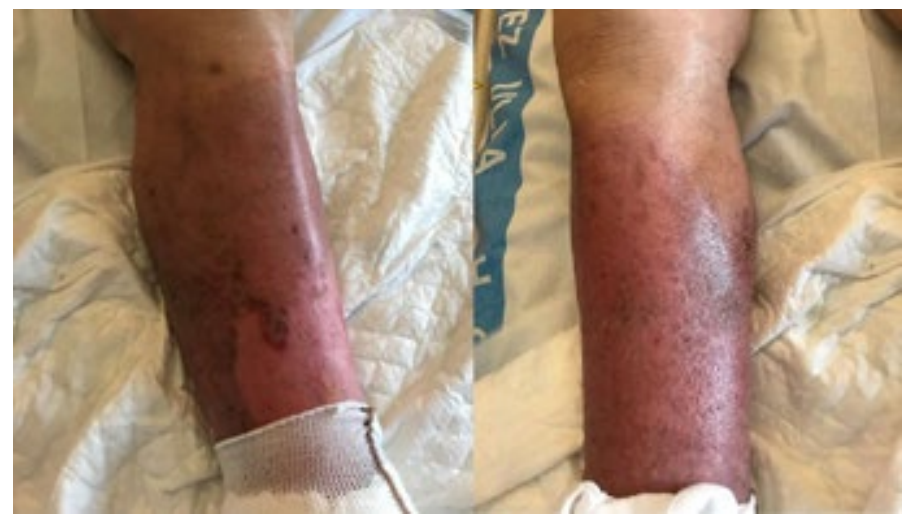

Figura 4. Aspecto de las lesiones de la paciente antes de recibir el alta.

La elefantiasis es una patología que se produce por ataques inflamatorios repetidos, con fibrosis y esclerosis de la piel y del tejido celular subcutáneo en la que ésta presenta cambios tróficos, tales como depósitos de grasa, acantosis y crecimientos verrugosos 9 .

En concreto, la ENV es el grado más avanzado del linfedema crónico no filariásico. La acumulación continuada de líquido intersticial induce una proliferación de fibroblastos afectando a la respuesta inmune local, lo que conduce a una mayor fibrosis de la dermis y a un aumento de la susceptibilidad a infecciones ${ }^{10}$. Se caracteriza por presentar una piel con hiperqueratosis, papi- 
lomatosis e hiperplasia verrucosa, lo que le da un aspecto «en empedrado», pudiendo dar lugar a ulceración de las lesiones e incluso infección por bacterias y hongos. El diagnóstico es clínico y para llegar a él debemos descartar otras patologías que producen aumento de volumen de las extremidades como la dermatitis por estasis venosa, la cromoblastomicosis, la papilomatosis cutis carcinoide, el síndrome Stewart-Treves, la mucinosis papular, el mixedema pretibial y el lipedema ${ }^{10}$.

La elefantiasis trópica o filariasis es la forma más común de elefansiasis, como resultado de un bloqueo linfático por vermes parasitarios endémicos de áreas tropicales y subtropicales ${ }^{11}$. Subtipos de elefantiasis no filariásica incluyen los casos congénitos hereditarios asociados a la enfermedad de Milroy y la ENV que engloba los casos de linfedema crónico no filariásico tanto de causa infecciosa como no infecciosa ${ }^{12}$.

En nuestro caso, la causa fundamental del desarrollo de linfedema fue el estasis venoso. La paciente, según se averiguó durante su ingreso, padecía un proceso depresivo que la llevaba a permanecer la mayor parte del día postrada en la cama. Este hecho ha sido descrito en otros trabajos como causa predisponente para el desarrollo de ENV ya que no es habitual que una persona sana sea capaz de permitir que las lesiones evolucionen tanto como para que se produzca la $\mathrm{ENV}^{13}$.

Los casos de miasis por musca doméstica en pacientes con ENV se han descrito en la bibliografía encontrándose ejemplos en los que el estado psiquiátrico del paciente le lleva a que estos insectos aniden en los surcos creados por la hiperqueratosis $^{14,15}$.

El diagnóstico de estas miasis y su extensión a los tejidos blandos circundantes puede ser llevado a cabo a través del uso del ecógrafo ${ }^{16}$, aunque se puede llegar al diagnóstico con una exploración exhaustiva de los surcos que presentan las lesiones (Figura 2).

El ATC es un agente exfoliante ampliamente utilizado en multitud de patologías de la piel. En bajas concentraciones, el ATC es una preparación segura y ampliamente usada en peelings superficiales ${ }^{17}$.

El ATC es un compuesto inorgánico que provoca una desnaturalización proteica celular produciendo una quemadura, pero sin un gran efecto a nivel sistémico. A medida que el ATC penetra a través de la piel, provoca la necrosis coagulativa de las células en la epidermis y la dermis, así como de los vasos sanguíneos.

Conviene destacar que el empleo del ATC debe estar reservado a casos graves de ENV. Durante su uso, el paciente debe recibir una constante supervisión, ya que el uso inadecuado del ATC podría provocar daños importantes. Esto se debe a que el ATC es un agente autoneutralizante, de modo que cuando se aplica el ATC sobre la piel, éste seguirá penetrando hasta que se haya producido la coagulación de una cierta cantidad de proteína $^{18}$.

\section{CONCLUSIONES}

Bajo nuestro punto de vista, el uso del ATC en la ENV es una opción segura y asequible para el tratamiento de la hiperqueratosis. Si bien este uso del ATC no había sido descrito anteriormente, consideramos que se necesitan trabajos más exhaustivos para determinar la eficacia, las indicaciones y los límites del uso del ATC en patologías como la ENV.

\section{BIBLIOGRAFÍA}

1. Kar Keong N, Siing Ngi AT, Muniandy P, Voon Fei W. Elephantiasis nostras verrucosa: a rare complication of lower limb lymphoedema. BMJ Case Rep. 2017: bcr2017221492.

2. Grada AA, Phillips TJ. Lymphedema: Pathophysiology and clinical manifestations. J Am Acad Dermatol. 2017; 77(6): 1009-20.

3. Manupati S, Ahmed FZ. Elephantiasis nostras verrucosa secondary to congestive cardiac failure. BMJ Case Rep. 2016 4; 2016: bcr2016214720.

4. Pitcher AA, Pagan CA, Small K, Otterburn DM. Excision of Elephantiasis Nostras Verrucosa Lesions in a Patient With Hereditary Lymphedema: Case Report and Review of the Literature. J Foot Ankle Surg. 2015; 54(4): 747-50.

5. Eda Y, Arita Y, Ogasawara N, Hasegawa S. Tolvaptan for the Treatment of Elephantiasis Nostras Verrucosa. Interna Medicine Tokyo. 2019; 58(22): 3347-8.

6. Sancho ADQ, Cura LRD, Alonso SA, López MAG. Treatment of Elephantiasis Nostras Verrucosa with CO2 Laser. Indian Dermatol Online J. 2019; 10(6): 704-6.

7. Hajdu SI. Elephantiasis. Ann Clin Lab Sci. 2002; 32(2): 207-9.

8. Halsted W. The swelling of the arm after operation for cancer of the breast. Elephantiasis chirurgic. Its cause and prevention. Bull Johns Hopkins Hosp. 1921; 32: 309-313.

9. Baird D, Bode D, Akers T, Deyoung Z. Elephantiasis Nostras Verrucosa (ENV): a complication of congestive heart failure and obesity. J Am Board Fam Med. 2010; 23(3): 413-7.

10. Tiwari A, Cheng K-S, Button M, Myint F, Hamilton G. Differential diagnosis, investigation, and current treatment of lower limb lymphedema. Arch Surg.2003; 138(2): 152-61.

11. Lourens GB, Ferrell DK. Lymphatic Filariasis. Nurs Clin North Am. 2019; 54(2): 181-92.

12. Lee KR, Bershow A, Crowson AN. Elephantiasis nostras verrucosa secondary to scleroderma. Cutis. 2019; 103(2): E4-6.

13. Simón Llanes J, Coll Vilar I, Tamarit Francés C, Niubó de Castro I. Elefantiasis verrucosa nostras en paciente afectado de trastorno depresivo mayor [Elephantiasis nostras verrucosa in a $\mathrm{p}$ [atient with major depressive disorder]. Semergen. 2012; 38(8): 526-9.

14. Ferreira-González L, Vázquez Vázquez B, García Alén D, Gómez Gigirey A. Elefantiasis verrucosa nostra colonizada. Galicia Clin 2012; 73(4): 173-17.

15. Borst GM, Goettler CE, Kachare SD, Sherman RA. Maggot Therapy for Elephantiasis Nostras Verrucosa Reveals New Applications and New Complications: A Case Report. Int J Low Extrem Wounds. 2014; 13(2): 135-9.

16. Nicholas E, Gaskin K, Wojcik S. Sonographic Detection of Cutaneous Myiasis. Clin Pract Cases Emerg Med. 2019; 3(4): 438-9.

17. Truchuelo M, Cerdá P, Fernández LF. Chemical Peeling: A Useful Tool in the Office. Actas Dermosifiliogr. 2017;108(4): 315-22.

18. Vanhooteghem O, Henrijean A, Devillers C, Delattre L, de la Brassinne M. La technique du peeling à l'acide trichloracétique. Mode d'emploi et précautions [Trichloracetic acid peeling: method and precautions]. Ann Dermatol Venereol. 2008; 135(3): 239-44. 